\title{
Doppler Boosting Effect on the Jet Radiation of Gamma-Ray Bursts and Active Galactic Nuclei
}

\author{
Xiao-Li Huang, Hai-Ming Zhang, Shu-Qing Zhong and En-Wei Liang \\ Guangxi Key Laboratory for Relativistic Astrophysics, Department of Physics, Guangxi \\ University, Nanning 530004, China \\ email: lew@gxu.edu.cn
}

\begin{abstract}
High energy photon radiations of gamma-ray bursts (GRBs) and active galactic nuclei $(\mathrm{AGNs})$ are dominated by their jet radiations. It was suggested that relativistic jets powered by different mass-scale black holes may share the same physical laws. A tight relation among the peak luminosity, the peak photon energy in the $\nu f_{\nu}$ spectrum, and the initial Lorentz factor is found for GRBs. With samples of GeV-TeV BL Lacs, FSRQs, and NLS1 galaxies, we show that these sources do not follow this relation. This may be attributed to the jet geometry and continuous/episodic jet as well as radiation physics for different kinds of sources.
\end{abstract}

Keywords. Gamma-Ray Bursts; Active Galactic Nuclei; Relativistic Jets; Non-thermal Radiation

\section{Introduction}

It is believed that gamma-ray bursts (GRBs) are produced by an ultra-relativistic jet powered by stellar black holes (BHs) from core collapses of massive stars or mergers of two compact stars (e.g., Kumar \& Zhang 2015), and the high energy photon radiations of active galactic nuclei (AGNs) are dominated by radiations from a mildly relativistic jet fed by accretion of their central super-massive BHs (e.g. Ghisellini et al. 2009; Zhang et al. 2012). Some comparative studies show evidence of similar jet properties between AGNs and GRBs (e.g., Wang et al. 2011; Nemmen et al. 2012; Zhang et al. 2013). Liang et al. (2015) found a tight correlation among the peak luminosity $\left(L_{\mathrm{p}}\right)$, the peak energy $\left(E_{\mathrm{p}, \mathrm{z}}\right)$ of the $\nu f_{\nu}$ spectrum in the GRBs rest frame, and the initial Lorentz factor $\left(\Gamma_{0}\right)$ of GRBs jets. This paper investigates whether or not the synchrotron radiations from AGNs jets follow the same relation with GRBs jets.

\section{Data}

Zhang et al. $(2012,2015)$ and Sun et al. (2015) derived the jet properties of GeV-TeV selected BL Lacs, flat spectrum radio quasars (FSRQs), and narrow-line Seyfert 1 (NLS1) galaxies by fitting their SEDs with the lepton models. We collected the peak luminosity $\left(L_{\mathrm{syn}}\right)$ and photon energy $\left(E_{\mathrm{syn}, \mathrm{z}}\right)$ of the synchrotron radiations and the Doppler boosting factors $(\delta)$ of these objects from their analysis. Since they assumed $\delta=2 \Gamma$ in their fits, where $\Gamma$ is the bulk Lorentz factor of the jets, we calculate the $\Gamma$ values with $\Gamma=\delta / 2$.

\section{Results}

Firstly, we test if the synchrotron radiations of the AGNs follow the $L_{p}-E_{p, z}-\Gamma_{0}$ relation of GRBs, i.e., $L_{p, 52} \propto E_{p, z}^{1.34 \pm 0.14} \Gamma_{0}^{1.32 \pm 0.19}$ (Liang et al. 2015). We calculate the 

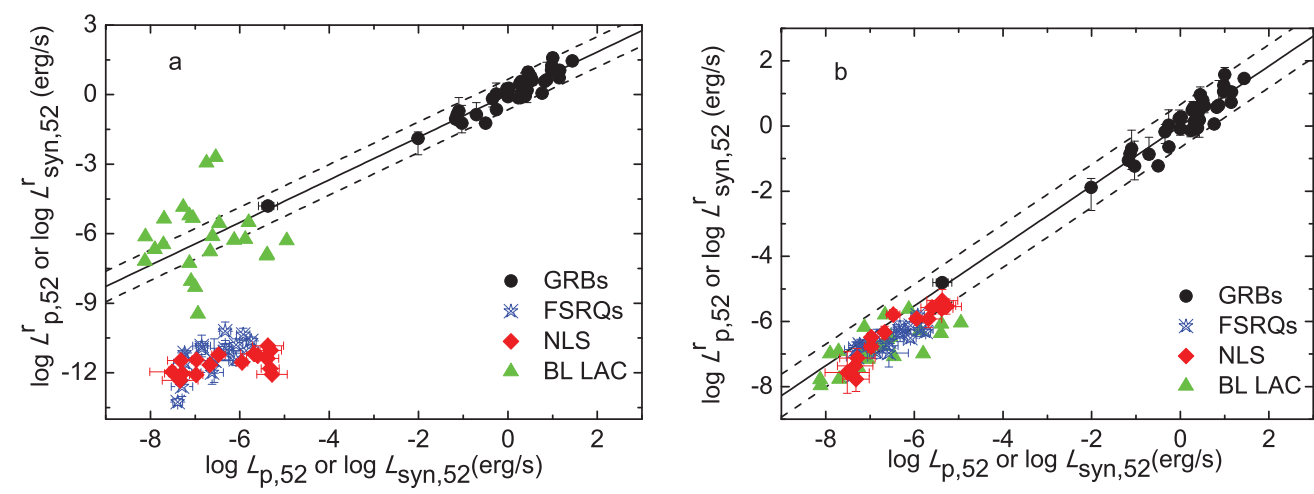

Figure 1. Distributions of the AGNs in the $\log L_{\mathrm{p}}^{\mathrm{r}}-\log L_{\mathrm{p}}$ plane, where $\log L_{\mathrm{p}}^{\mathrm{r}}$ is calculated by assuming that the AGNs follow the $L_{p}-E_{p, z}-\Gamma_{0}$ relation of GRBs (panel a; the solid and dashed lines; Liang et al. 2015) or by taking the relations for different type AGNs (panel b).

synchrotron peak luminosity $\left(L_{\mathrm{syn}}^{\mathrm{r}}\right)$ for these AGNs with this relation by using their $E_{\mathrm{syn}, \mathrm{z}}$ and $\Gamma$. Figure $1(\mathrm{a})$ shows $L_{\mathrm{syn}}^{\mathrm{r}}$ as a function of $L_{\mathrm{syn}}$ in comparison with the GRBs from Liang et al. (2015). It is found that BL Lac objects are distributed at the low $L_{\mathrm{p}}$ end of this relation, but the scatter is very large. Both FSRQs and NLS1 galaxies, on the other hand, heavily deviate from this relation. We then separately make multiple regression analysis to the BL Lacs, FSRQs and NLS1 galaxies to derive their $L_{\mathrm{syn}}^{\mathrm{r}}\left(E_{\mathrm{syn}, \mathrm{z}}, \Gamma\right)$ relations, and obtain $L_{\mathrm{syn}, 52} \propto E_{\mathrm{syn}, \mathrm{z}}^{0.01 \pm 0.01} \Gamma^{2.35 \pm 0.52}, L_{\mathrm{syn}, 52} \propto E_{\mathrm{syn}, \mathrm{z}}^{0.57 \pm 0.15} \Gamma^{2.58 \pm 0.81}$, and $L_{\text {syn }, 52} \propto E_{\mathrm{syn}, \mathrm{z}}^{0.51 \pm 0.27} \Gamma^{4.07 \pm 0.39}$, respectively. Figure $1(\mathrm{~b})$ shows the AGNs and GRBs in the $L_{\mathrm{p}}^{\mathrm{r}}-L_{\mathrm{p}}$ plane by using their $L_{\mathrm{syn}}^{\mathrm{r}}\left(E_{\mathrm{syn}, \mathrm{z}}, \Gamma\right)$ relations for different types of sources.

\section{Conclusions}

We have shown that the AGNs jet radiation does not follow the same $L_{\mathrm{syn}}^{\mathrm{r}}\left(E_{\mathrm{syn}, \mathrm{z}}, \Gamma\right)$ relations as the $L_{\mathrm{p}, \mathrm{z}}^{\mathrm{r}}\left(E_{\mathrm{p}, \mathrm{z}}, \Gamma_{0}\right)$ relation of GRBs. The dependence of the luminosity on the Lorentz factor is dramatically different among these sources. This may be due to different jet geometry and continuous/episodic jets (e.g., Ghisellini et al. 1993). The different dependence of the luminosity on $E_{\mathrm{p}, \mathrm{z}}$ may hint at different radiation physics among these sources (e.g., Lyu et al.2014).

This work is supported by the National Basic Research Program of China (973 Program, No. 2014CB845800), the National Natural Science Foundation of China (No. 11533003), the Guangxi Science Foundation (No. 2013GXNSFFA019001). XLH was supported by an IAU travel grant.

\section{References}

Ghisellini, G., Padovani, P., Celotti, A., \& Maraschi, L., 1993, ApJ, 407, 65

Ghisellini, G., Tavecchio, F., \& Ghirlanda, G., 2009, MNRAS, 399, 2041

Kumar, P. \& Zhang, B., 2015, PhR, 561, 1

Liang, E.-W., Lin, T.-T., Lü, J., Lu, R.-J., Zhang, J., \& Zhang, B., 2015, ApJ, 813, 116

Lyu, F., Liang, E.-W., Liang, Y.-F., Wu, X.-F., et al.2014, ApJ, 793, 36

Nemmen, R. S., Georganopoulos, M., Guiriec, S., et al. 2012, Sci, 338, 1445

Sun, X.-N., Zhang, J., Lin, D.-B., Xue, Z.-W., Liang, E.-W., \& Zhang, S.-N., 2015, ApJ, 798, 43 
Wang, J. \& Wei, J. Y., 2011, ApJ, 726, L4

Zhang, J., Liang, E.-W., Sun, X.-N., Zhang, B., Lu, Y., \& Zhang, S.-N., 2013, ApJ, 774, L5

Zhang, J., Liang, E.-W., Zhang, S.-N., \& Bai, J. M., 2012, ApJ, 752, 157

Zhang, J., Xue, Z.-W., He, J.-J., Liang, E.-W., \& Zhang, S.-N., 2015, ApJ, 807, 51 